\title{
Challenges in Health Smart Homes
}

\author{
Paulo Bartolomeu \\ Micro I/O \\ Aveiro, Portugal \\ bartolomeu@microio.pt
}

\author{
José Fonseca \\ DETI-UA \\ Aveiro, Portugal \\ jaf@ua.pt
}

\author{
Francisco Vasques \\ DEMEGI-FEUP \\ Porto, Portugal \\ vasques@fe.up.pt
}

\begin{abstract}
This paper presents an overview of the challenges in developing Health Smart Homes targeting, in particular, elderly and impaired people. As such, it identifies the current endeavors as well as the main areas of research.
\end{abstract}

Keywords- smart homes; assistive technologies; design for all; reliability; security; networking

\section{INTRODUCTION}

The world population aged over 65 years is expected to grow above 1490 million persons by 2050 [1]. According to this forecast, by the end of the current half century, Asia alone will contribute with over 923 millions while Europe and America add 183 and 238 millions, respectively. The relative elderly population is expected to grow on par with the increase of average life expectancy fueled by technical improvements in healthcare services and technology [2]. Given the expected impact on health and social services, solutions should be investigated to mitigate the resulting effects.

Dishman [3] identifies four requirements that should be addressed to cope with the current trends of elderly demographic increase: promote healthy behaviors, detect diseases in an early stage, improve treatment compliance and provide support for informal caregiving. These requirements can be met by maintaining elderly (or impaired) people living in their homes [4], within their social network, and being assisted by automation systems that, besides helping them with the Activities of Daily Living (ADLs) also monitor their health condition (e.g. vital parameters, advanced chemical analysis, etc.).

This work aims at identifying the key constraints in developing Health Smart Homes targeting elderly and impaired people, the areas where further investigation is required and the challenges they provide.

The paper is organized as follows: section 2 presents a survey on Smart Homes by focusing current research projects addressing those that deal with the support of health services. Section 3 identifies the main research areas in this domain as well as the associated challenges. Section 4 presents the BLive $^{\circledR}$ system by focusing on the current efforts being developed. This paper is concluded with an overview of its findings.

This work was supported by Fundação para a Ciência e a Tecnologia under grant POSI 2004 Medida 6.1, Ref. SFRH/BDE/15602/2006 and by Micro I/O - Serviços de Electrónica, Lda.

\section{SMART HOMES}

In [5] Jiang et al. presented the Intertek [6] concept of Smart Home (SH) as a "...dwelling incorporating a communications network, that connects the key electrical appliances and services, and allows them to be remotely controlled, monitored or accessed...". This field emerged from the research activity in both home automation [7] and home networking areas [8]. The inclusion of Assistive Technologies altogether with the requirements of elderly and impaired users drove the assistive social-oriented application of Smart Houses [9] into the health domain, originating the concept of Health Smart Home (HSH) [6].

A large set of research projects in the field Smart Homes has been identified in [5], including research within the academia (KTH's comHOME, MIT's House of the Future and the University of Massachusetts Intelligent Home, just to mention some) and within the industry (e.g. Cisco's Internet Home, Microsoft's EasyLiving, Siemens Smart Home and Intel Architecture Labs). Despite the intense activity on this area of research, only two of these projects explicitly address the requirements of elderly or impaired people: CUSTODIAN and Portsmouth Smart Home. The CUSTODIAN project has been developed to "create barrier-free homes for homeowners with disabilities" while the Portsmouth Smart Home was designed to "support independent living for disabled users".

In [6] Noury et al. defined the Health Smart Home as being aimed "... at giving an autonomous life, in their own home, to people who would normally be placed in institutions...". The authors also provide a state of the art on health smart homes going back to 1998 where allegedly it all started with the monitoring of daily life activities and physiological parameters.

Currently, efforts are being developed in designing inclusive HSHs that cope with the requirements of elderly and impaired people. These efforts resulted in some noteworthy endeavors such as partner consortiums (MonAMI [10]), interdisciplinary research groups (Smart Medical Home [11], Aware Home [12], TAFETA [13]), in-home laboratories (Gator Tech House [14], Duke Smart Home [15]) and retrofitting and adaptation solutions (B-Live ${ }^{\circledR}$ system [16]).

Current research has evolved from the point where developments were essentially technical to a stage where social 
aspects are also taken into account. The next section identifies the open challenges in HSHs.

\section{Challenges In Heath SMart Homes}

As pointed, several endeavors are being developed to build effective HSHs. However, the majority is in an early stage of development and thus far from being widely disseminated in common dwellings.

The design of HSHs is complex and time-consuming, as a large set of requirements from formal and informal occupants must be identified, studied and validated. Dewsbury et al. [17] argue that "...the required characteristics of the assistive technology should be considered under four headings": fitness of purpose, trustworthiness, acceptability and adaptability.

The fitness for purpose heading states that systems should work according to the user's desires, expectations and requirements, in an intuitive and transparent fashion.

The trustworthiness heading includes requirements related with dependability, as defined by Laprie [18] (Availability and Reliability, Safety, Maintainability, Confidentiality and Integrity).

Acceptability aggregates requirements associated with characteristics that contribute to the user's acceptance of the system such as Usability, Learnability, Affordability, Compatibility, Efficiency, Responsiveness and Aesthetics.

Adaptability requirements are related with the environment in the home as well as the stakeholders of the system. This heading is intimately associated with the ability to modify domestic systems by adding, configuring or reconfiguring equipment. Therefore, it includes requirements such as Configurability, Openness, Visibility and User reparability, which can be systematically assessed using the Dewsbury Method for Dependable Domestic Systems (MDDS) [19].

Although providing a systematical approach to collect and analyze the user's requirements, this method significantly relies on the interviewer's ability to infer requirements from the provided checklists. As such, it lacks the metrics to make it less prone to human misjudgment and time-consuming validation. The following sub-sections explore representative on-going research activities in key areas of HSHs.

\section{A. Design for All}

Early smart homes were designed to provide comfort to standard users by supporting the control of environment variables (lighting, temperature, ...) and a limited range of appliances such as doors and blinds. Nowadays, the design philosophy has shifted towards an approach commonly known as design for all which has a "multidisciplinary connotation, and refers to the design of interactive products, services, and applications that are suitable for most potential users without any modifications" [20]. As such, in order to improve the universal accessibility of these systems, all potential HSH resident's requirements should be accounted, in the design phase, at biological, psychological and social levels [21].
The usability of HSHs is a major concern when designing for all. Therefore, efforts should be made to guarantee interfaces easily learnable, efficient, memo-rable, resilient to errors that satisfy the stakeholders [22].

\section{B. Security and Privacy}

Security and privacy are two critical concerns in the field of developing Smart Homes, not only for the direct implications of their violation but also because their support, in devices designed for elderly and impaired people, is an open research area. Furthermore, wireless communications are becoming very popular among home networking applications given their low installation cost, aesthetics, flexibility and reconfiguration capabilities [23]. However, wireless communications are more exposed to security and privacy breaches than its wired counterparts. According to Bergstrom et al., to ensure security, a network must provide confidentiality, authentication, authorization and integrity [24]. Consequently, the development of a HSH must be based on a network design that meets these requirements.

Another important aspect is privacy, not only from the trust perspective [25] but also from an ethical point of view [26]. For example, the use of video (e.g. AT\&T Home Monitoring Service [27]) or data mining (e.g. QuietCare ${ }^{\circledR}$ [28]) technologies for tracking elderly or impaired people activity can have a positive impact on their wellness by increasing the perceived sense of freedom. However, without adequate privacy regulation, users are unprotected from abuses. Efforts should then be made in developing adequate regulation to protect the user's privacy as well as maintaining the benefits of using technology to enhance wellness.

\section{Reliability and Safety}

The support of safety in Smart Homes is a domain often regarded as being mainly related with security and protection of privacy. However, it is dependent on a broader spectrum of scenarios [29]: system misuse, software bugs, hardware malfunctions, interference on communications, etc.

If a Smart Home user has the ability to perform a set of actions or commands that require human intervention for completion or continuous operation, he/she may utilize the system improperly and cause damages not only in the House but also to him/herself or someone in the neighborhood.

Another scenario arises from software bugs or hardware malfunctions. In this case the system has no predictable behavior and can damage equipment or harm occupants. As most domotic systems use buses for communicating data, there is also the possibility of having the system working on unpredictable states due to malfunction of the network caused by interference from appliances (e.g. micro oven).

So, because Smart Homes must ensure the safety of stakeholders and equipments, designers must envision ways of avoid software, hardware and network malfunctions as well as mechanisms to actuate on their occurrence to mitigate possible harmful consequences. 


\section{Ambient Intelligence}

"The objective of Ambient Intelligence (AmI) is to broaden the interaction between human beings and digital information technology through the usage of ubiquitous computing devices" [20]. The inclusion of AmI in the home aims at improving the quality of life of residents, for example increasing the autonomy of elderly or impaired people in performing ADLs [30].

AmI should follow high-level design requirements such as being unobtrusive, personalized, adaptive and anticipatory. These can be met by embedding intelligent and intuitive interfaces in all types of home objects and by having embedded devices collaborating to improve the quality of life of residents, for example, by automating tasks and providing context aware functionalities.

\section{E. Networking}

Several wired technologies (KNX, LonWorks, CEBus, $\mathrm{X} 10$, etc.) address the problematic of supporting data communication in Smart Homes. However, factors such as the cost decrease in electronics and the consumer demand for seamless and effortless access to services are fueling the emergence of wireless communications in the Smart Home domain. In consequence, efforts are being made in different, yet complementary, directions such as augmenting standard wired technologies (e.g. KNX RF [23]), enhancing standard wireless technologies (e.g. IEEE 802.11e [31]) and developing solutions based on emergent wireless technologies and protocols (e.g. Wireless Mesh [32] and Sensor [33] networks).

As introduced, the design of Smart Homes must cope with requirements in order to become fit for purpose, trustworthy, acceptable and adaptable. From a performance perspective, these are Availability, Reliability and Responsiveness. In consequence, the adoption of wireless technologies depends of the ability to support real-time communications and faulttolerance mechanisms capable of meeting the HSHs' requirements.

\section{IMPLEMENTING WIRELESS HSHS}

The B-Live ${ }^{\circledR}$ system is a flexible and modular home automation solution that enables conventional homes to become HSHs. It was developed to address the requirements of elderly and impaired people and to promote the dissemination of low cost assistive technologies. Besides enabling the control of common house appliances (e.g. lights, doors, window blinds, etc.) using standard or customized (e.g. vocalized menu) interfaces, it also supports the integration of health sub-systems. Currently, low tract urinary diseases can be monitored using the B-Live ${ }^{\circledR}$ uroflowmeter prototype.

The B-Live ${ }^{\circledR}$ system was originally designed with CAN [34], a popular fieldbus pushed by the automotive industry due to its low cost and support for timeliness [35] and faulttolerance [36]. Currently, we are evaluating the feasibility of replacing this technology by a wireless counterpart based on the emergent IEEE802.15.4 [37] protocol. The impact on reliability, security, timeliness and fault tolerance is being evaluated using a broadcast approach to seamless substitute the CAN fieldbus. Hybrid solutions may also be considered in safety-critical applications that can cope with the installation of physical wires.

Although communications play an important role in the BLive $^{\circledR}$ system's trustworthiness, other aspects such as fitness of purpose, acceptability and adaptability must be met in order to make it appealing for the target users. Originally, the B-Live ${ }^{\circledR}$ system was developed envisaging a particular application within the "Centro de Medicinal e Reabilitação da Região Centro - Rovisco Pais" (CMRRC-RP), a Portuguese rehabilitation center, where an apartment was enhanced to cope with the requirements of tetraplegic and paraplegic patients. Nowadays, we recognize that this endeavor would benefit the general population, in particular elderly and impaired people and, as such, efforts are being made to extend the B-Live ${ }^{\circledR}$ system's functionality and usability to make it suited for all.

\section{CONCLUSION}

This paper presented an overview of the current endeavors in Health Smart Homes as well as the challenges they provide. In addition, the B-Live ${ }^{\circledR}$ system was introduced as a retrofitting solution that enables common dwellings to cope with the requirements of elderly and impaired people. Currently, this system is being enhanced to support healthcare services and wireless communications.

\section{REFERENCES}

[1] Division of the Department of Economic and Social Affairs of the United Nations Secretariat, "Growth of the Europe population", World Population Prospects: The 2006 Revision and World Urbanization, 2006.

[2] Coughlin, J., "Technology needs of aging boomers", Issues in Science and Technology, vol. 16, no.1, pp.53-60, 1999.

[3] Dishman, E., "Inventing wellness systems for aging in place", Computer Journal, Vol. 37, Issue 5, pp. 34-41, May 2004.

[4] Harmo, P., Taipalus, T., Knuuttila, J., Vallet, J., Halme, A., "Needs and Solutions - Home Automation and Service Robots for the Elderly and Disabled," Proceedings of the IEEE/RSJ International Conference on Intelligent Robots and Systems (IROS 2005), Edmonton Canada, August 2005.

[5] Jiang, L., Liu, D., Yang, B., "Smart home research", Proceedings of the 2004 International Conference on Machine Learning and Cybernetics, Vol. 2, pp. 659- 663, August 2004.

[6] Noury, N., Virone, G., Barralon, P., Ye, J., Rialle, V., Demongeot, J. "New trends in health smart homes", Proceedings of the 5th International Workshop on Enterprise Networking and Computing in Healthcare Industry (Healthcom 2003), pp. 118 - 127, June 2003.

[7] Gutierrez, J.A., "On the use of IEEE 802.15.4 to enable wireless sensor networks in building automation," Proceedings of the 15th IEEE International Symposium on Personal, Indoor and Mobile Radio Communications (PIMRC 2004), Vol. 3, pp. 1865-1869, September 2004.

[8] Kastner, W., Neugschwandtner, G., Soucek, S., Newmann, H.M., "Communication Systems for Building Automation and Control", Proceedings of the IEEE, Vol. 93, No. 6, pp. 1178- 1203, June 2005.

[9] Stefanov, D.H., Bien, Z., Bang, W., "The smart house for older persons and persons with physical disabilities: structure, technology arrangements, perspectives", IEEE Transactions on Neural Systems and Rehabilitation Engineering, Vol. 12, I2, pp. 228-250, June 2004. 
[10] Mainstreaming on Ambient Intelligence (MonAMI) project, http://www.monami.info, 2007.

[11] Smart Medical Home Research Laboratory, Center for Future Health, University of Rochester, http://www.futurehealth.rochester.edu, 2007.

[12] Kidd, C., Orr, R., Abowd, G., Atkeson, C., Essa, I., MacIntyre, B., Mynatt, E., Starner, T., Newstetter, W., "The Aware Home: A Living Laboratory for Ubiquitous Computing Research", Proceedings of the 2nd International Workshop on Cooperative Buildings, Integrating Information, Organization, and Architecture, pp. 191-198, October1999.

[13] Knoefel, F., Emerson, V., Schulman., B., "TAFETA: An Inclusive Design for Tele-Health", Technology and Persons with Disabilities Conference, March 2005.

[14] Helal, S., Mann, W., El-Zabadani, H., King, J., Kaddoura, Y., Jansen, E.. "The Gator Tech Smart House: A Programmable Pervasive Space", IEEE Computer, Vol. 38, No. 3, pp. 50-60, March 2005.

[15] Home Depot Smart Home, Pratt School of Engineering, Duke University, http://www.smarthome.duke.edu, July 2007.

[16] Bartolomeu, P., Fonseca, J., Santos, V., Mota, A., Silva, V., Sizenando M., "Automating Home Appliances for Elderly and Impaired People: The B-Live Approach", Proceedings of the 2nd International Conference on Software Development for Enhancing Accessibility and Fighting Info-exclusion (DSAI2007), November 2007.

[17] Dewsbury1, G., Rouncefield, M., Clarke, K., Sommerville, I., "Depending on digital design: extending inclusivity", Housing Studies, Vol. 19, No. 5, pp. 811-825, September 2004.

[18] Laprie, J.C., Avizienis, A., Kopetz, H., "Dependability: Basic Concepts and Terminology", Springer-Verlag New York, Inc., 1991.

[19] Dewsbury, G., "Method for Dependable Domestic Systems", http://www.smartthinking.ukideas.com/ MDDS.html, consulted in September 2007.

[20] Emiliani, L., Stephanidis, C., "Universal access to ambient intelligence environments: Opportunities and challenges for people with disabilities", IBM Systems Journal, Vol. 44, No. 3, pp. 605, 2005.

[21] Fisk, A., Rogers, W., Charness, N., Czaja, S., Sharit, J., "Designing for older adults: Principles and Creative Human Factors Approaches", CRC Press, February 2004.

[22] Gamberini, L., Alcaniz, M., Barresi, G., Fabregat, M., Ibanez, F., Prontu, L., "Cognition, technology and games for the elderly: An introduction to ELDERGAMES Project”, PsychNology Journal, Vol. 4, No. 3, pp. 285, 2006.

[23] Reinisch, C., Kastner, W., Neugschwandtner, G., Granzer, W., "Wireless Technologies in Home and Building Automation", Proceedings of the
International Conference on Industrial Informatics (INDIN2007), July 2007.

[24] Bergstrom, P., Driscoll, K., Kimball, J., "Making home automation communications secure”, IEEE Computer Magazine, Vol. 34, pp. 50-56, October 2001.

[25] Schaefer, R., Ziegler, M., Mueller, W., "Securing Personal Data in Smart Home Environments", Workshop on Privacy-Enhanced Personalization (PEP2006), 2006.

[26] Hoof, J., Kort, H., Markopoulos, P., Soede, M., “Ambient Intelligence, Ethics and Privacy," Gerontechnology Journal, Vol. 6, No. 3, pp. 155163, July 2007.

[27] AT\&T Home Monitoring Service, http://www.att.com/ gen/pressroom?pid=8595\#, Sept. 2007.

[28] QuietCare - A home health security system, http://www.quietcare.com/, July 2007.

[29] Chetan, S., Ranganathan, A., Campbell, R., "Towards fault tolerance pervasive computing", IEEE Technology and Society Magazine, Vol. 24, Issue 1, pp. 38- 44, Spring 2005.

[30] Abascal, J., "Ambient Intelligence for people with disabilities and elderly people", Proceedings of the SIGCHI Workshop, 2004.

[31] Frikha, M., Said, F., Maalej, L., Tabbana, F., "Enhancing IEEE 802.11e standard in congested environments", Proceedings of the International Conference on Telecommunications and on internet and Web Applications and Services, February 2006.

[32] Akyildiz I.F., Wang, X., "A Survey on Wireless Mesh Networks", IEEE Communications Magazine, Vol. 43, No. 9, pp. S23- S30, September 2005.

[33] Callaway, E., "Wireless Sensor Networks: Architectures and protocols", CRC Press, 2004.

[34] Bosch, "Controller Area Network (CAN) specification - version 2.0, part A", Bosch GmbH, Germany, 1991.

[35] Almeida, L., Pedreiras, P., Fonseca, J., "The FTT-CAN Protocol: Why and How," IEEE Transactions on Industrial Electronics, Vol. 49, No. 6, December 2002.

[36] Ferreira, J., Almeida, L., Fonseca, A., Pedreiras, P., Martins, E., Rodriguez-Navas, G., Rigo, J., Proenza, J., "Combining Operational Flexibility and Dependability in FTT-CAN", IEEE Transactions on Industrial Informatics, Vol. 2, Issue 2, pp.95 - 102, May 2006.

[37] IEEE Std. 802.15.4 - Standard for Information technology, September 2006. 